Pacific Journal of Mathematics

A COMPOSITE NEWTON-RAPHSON GRADIENT METHOD 


\title{
A COMPOSITE NEWTON-RAPHSON GRADIENT METHOD FOR THE SOLUTION OF SYSTEMS OF EQUATIONS
}

\author{
William L. HaRT AND TheOdoRe S. MOTZKIN
}

1. Introduction. This article was motivated by the desire to obtain an iterative method for solving a system of equations, linear or not, into which all equations would enter symmetrically, and which would be suitable for numerical application, particularly on a high speed digital computing machine.

The general problem considered is the solution of a system of $k$ equations $\left\{f_{j}(x)=0\right\}$ in $n$ unknowns $\left(x_{1}, \cdots, x_{n}\right)=x$ where, as throughout the paper, all variables and function values are real. Each step of our method consists in obtaining, from one approximation $x$ to a solution of the system, the next approximation by adding to $x$ the vector sum of corrections parallel to the gradients of the $k$ functions $f_{j}(x)$. The lengths of the corrections are regulated by individual weights and by use of a factor $\rho \neq 0$. The component gradient correction for a single equation $f_{j}(x)=0$ is of the Newton-Raphson type because the correction, if applied to an initial approximation $x^{(0)}$, gives a point annihilating the usual linear approximation to $f_{j}(x)$ for $x$ near $x^{(0)}$.

After considering in $\S 2$ the well known formula for a gradient correction to an approximate solution $x^{(0)}$ of a single equation $f(x)=0$, the method of composite gradient corrections for a general system is described in $\S 3$. In $\S 4$, we apply the method to a system of $k$ linear equations in $n$ unknowns, and prove that, for an arbitrary approximation $x^{(0)}$ to a solution of the system, we obtain a sequence $\left\{x^{(m)}\right\}$ which tends with a geometric rate of convergence to a point $\hat{x}$, nearest to $x^{(0)}$, of the set which satisfies the system in a sense of weighted least squares. Section 5 treats a fairly general system with an isolated solution $\hat{x}$. The sequence $\left\{x^{(m)}\right\}$ of $\S 3$ is proved to converge to $\hat{x}$ if the initial approximation $x^{(0)}$ is sufficiently near $\hat{x}$. Section 6 considers the implicit function $x=x(t)$ defined by a related system of $n$ equations $f(x$; $\tau)=0$, where $\tau=\left(\tau_{1}, \cdots, \tau_{k}\right)$, and $\tau=\tau(t), 0 \leqq t \leqq 1$. It is proved that, if $0=t_{0}<t_{1}<\cdots<t_{l}=1$ is a fine enough partition of the $t$-interval, then the sequence $\left\{x^{(m)}\right\}$ of $\S 3$ tends to $x\left(t_{i}\right)$ if $x^{(0)}=x\left(t_{i-1}\right)$. This result yields a small arc method for computing the points $x\left(t_{i}\right)$ in sequence.

There is an extensive literature on the solution of linear systems $\left\{f_{j}(x)=0\right\}$ by iterative processes where each iteration involves a correction related to a specified direction, in particular that of some gradient;

Received October 7, 1955. Presented to the American Mathematical Society, April 30, 1955. This work was sponsored (in part) by the Office of Ordnance Research, U.S. Army. 
see $[2$, p. 310]. Frequently, the correction involves preliminary minimization of a single function $g(x) \geqq 0$ built up from the $f_{j}$. A general method of this type is due to Hestenes and Stein, reference 53 in [2]. For an initial approximation $x$ and assigned direction $d$, they introduce a constant $\alpha^{*}$ such that $g(x+\alpha d)$ attains its minimum at $\alpha=\alpha^{*}$; the correction to $x$ is $\beta \alpha^{*} d$, where $\beta$ is a constant; $d$ and $\beta$ are subject to change at each iteration. This method could be specialized to the situation in our Theorem 4.1, where the correction to any $x$ is $\rho \Delta x=$ $\operatorname{grad}\left[\frac{1}{2} \rho g(x)\right]$, with $g(x)$ in (4.12). However, for simplicity in $\S 4$, and efficiency in its application to nonlinear systems in $\S \S 5$ and 6 , we have based $\S 4$ directly on $\S 3$, without using $g(x)$. For nonlinear systems, our correction to $x$ in general does not have the direction of the gradient of any single function $g(x)^{1}$.

A method of S. Kaczmarz for linear systems, reference 67 of [2], in common with our procedure, involves the gradients of the $f_{j}(x)$ separately. However, in contrast to the composite nature of our gradient corrections, he introduces corrections taken along the gradients of the $f_{j}$ in sequence, to carry out a single cycle of the iteration. For an arbitrary initial approximation $x^{(0)}$ to a solution, the Kaczmarz method may yield a sequence $\left\{x^{(m)}\right\}$ which is not convergent and only stays bounded [4], whereas the sequence $\left\{x^{(m)}\right\}$ of $\S 4$ always has a unique limit.

There are intimate contacts between our procedure in the linear case and a method due to L. F. Richardson, reference 98 in [2], and later to R. von Mises and Hilda Pollaczek-Geiringer, reference 123 in [2]. For a system ${ }^{2} x H+b=0$, in matrix form, and an initial approximation, $x$, to a solution, Richardson specifies the next approximation $y=x$ $-\rho(x H+b)$. With a system $\left\{f_{j}(x) \equiv \sum_{i=1}^{n} x_{i} a_{i j}+b_{j}=0\right\}$, or $x A+b=0$, if the Richardson method is applied to a related system $x \mathscr{A} \mathscr{A}^{\prime}+b \mathscr{A}^{\prime}=0$ (see $\S 4$ ), the approximations $\left\{x^{(m)}\right\}, m>0$, starting with a given $x^{(0)}$, are those of $\S 4$. Then, also, the condition (4.25) on $\rho$ in $\S 4$ becomes a well known condition for convergence of the Richardson method (see $[2, \mathrm{p} .311])$.

${ }^{1}$ For $f_{j}(x)=\varphi_{j}\left(r_{j}(x)\right)$, where $r_{j}(x)$ is the distance of $x$ from a given linear subspace $\Lambda_{j}$ of arbitrary dimension, and $\varphi_{j}$ an arbitrary differentiable function of one variable, we have with $\Delta x=\sum_{j} \eta_{j} \Delta_{j} x$ as in (3.3) and (3.2) for $\xi=x, \Delta x=\operatorname{grad} \sum \eta_{j} \Psi_{j}\left(r_{j}{ }^{2}\right)$, where $d \Psi_{j}\left(r_{j^{2}}\right) /$ $d\left(r_{j}{ }^{2}\right)=-\varphi_{j}\left(r_{j}\right) /\left(2 r_{j} d \varphi_{j} / d r_{j}\right)$. On the other hand for example, for $f_{1}=x_{1}, f_{2}=x_{2}{ }^{2}+2 x_{3}{ }^{2}, \Delta x$ does not have the direction of a gradient, which would (for $n=3$ ) imply $D=0$, where $D$ is the determinant $|\Delta x, \partial \Delta x,| \partial x \mid$. Indeed we have $D=\sum_{j, k} \eta_{j} \eta_{k} D_{j k}, D_{j k}=\left|\Delta_{j} x, \partial \Delta_{k} x,\right| \partial x \mid=$ $f_{j} f_{k} w_{j}^{-2}\left|\operatorname{grad} f_{j}, \operatorname{grad} f_{k}, \operatorname{grad} w_{k}{ }^{-2}\right|$. Now for our $f_{1}$ and $f_{2}, D_{21}=0$ whereas $D_{12}=-32$ $x_{2} x_{3} f_{1} f_{2} w_{2}{ }^{-4} \neq 0$.

${ }^{2}$ Capital script or italic letters will represent matrices. The transpose of $A$ will be denoted by $A^{\prime}$. We shall treat $x$ as a one-rowed matrix. 
The Kaczmarz method for the solution of linear systems has led to the development of a corresponding sequential projection method for nonlinear systems, introduced by Tompkins [6]. The distinction between the method of Kaczmarz and that of $\S 4$ implies a similar difference between the method of Tompkins and that of $\S 5$.

In a paper by Chernoff and Crockett [1], an isolated maximum of a function $f(x)$ is determined, essentially, by solving the system $\left\{\partial f / \partial x_{i}\right.$ $=0\}$ by an iterative method involving the gradient of $f(x)$, in some metric. With their main hypothesis [1, p. 34], our Theorem 5.1 also provides a sequence tending to $\hat{x}$.

Extensions of the present paper are planned to inequalities and to equations in the complex field.

2. A fundamental gradient formula. Let $f\left(x_{1}, \cdots, x_{n}\right)=f(x)$ be a given function, and let $x^{(0)}$ be any assigned point in the neighborhood of which $f(x)$ is continuously differentiable. Visualize $x^{(0)}$ as an approximation to a solution of $f(x)=0$. Then let $\Delta x^{(0)}=\left(\Delta x_{1}^{(0)}, \cdots, \Delta x_{n}^{(0)}\right)$ be a vector correction for $x^{(0)}$ parallel to the gradient of $f(x)$ at $x^{(0)}$, with the following definition:

where

$$
\Delta x_{i}^{(0)}=-\frac{f\left(x^{(0)}\right) f_{i}\left(x^{(0)}\right)}{w^{2}\left(x^{(0)}\right)}, \quad(i=1,2, \cdots, n)
$$

$$
f_{i}(x)=\frac{\partial f(x)}{\partial x_{i}} \text { and } w^{2}(x)=\sum_{i=1}^{n} f_{i}^{2}(x) \text {, }
$$

and we assume that $w(x) \neq 0$ on the range for $x$.

Let $x^{(1)}=x^{(0)}+\Delta x^{(0)}$, and define

$$
\hat{f}(x)=f\left(x^{(0)}\right)+\sum_{i=1}^{n}\left(x_{i}-x_{i}^{(0)}\right) f_{i}\left(x^{(0)}\right) .
$$

Then it can be verified that $\hat{f}\left(x^{(1)}\right)=0$. That is, $x^{(1)}$ annihilates the usual linear approximation to $f(x)$ for points $x$ near $x^{(0)}$. In particular, if $f(x)$ is linear, then $f\left(x^{(1)}\right)=0$, and $x^{(1)}$ is the orthogonal projection of $x^{(0)}$ onto the hyperplane $f(x)=0$.

Note 2.1. Suppose that $f(x)$ is a polynomial of degree $k$ in $x_{1}, \cdots$, $x_{n}$, and let $\Lambda$ be the surface $f(x)=0$ in $x$-space. The linear polar for $\Lambda$ corresponding to a point $x^{(0)}$ can be defined as the hyperplane

$$
k f\left(x^{(0)}\right)+\sum_{i=1}^{n} f_{i}\left(x^{(0)}\right)\left(x_{i}-x_{i}^{(0)}\right)=0 .
$$

With $\Delta x^{(0)}$ given by $(2.1)$, let $x^{(2)}=x^{(0)}+k \Delta x^{(0)}$. Then it can be verified that $x=x^{(2)}$ satisfies (2.3). Hence, $x^{(1)}$ is one $k$ th of the way from $x^{(0)}$ 
to the polar hyperplane for $x^{(0)}$, along the normal from $x^{(0)}$ to this hyperplane.

It is important to recognize that the vector $\Delta x$, obtained from (2.1), is unaltered if $f(x)$ is changed to $c f(x)$, where $c$ is any nonzero constant. Also, with $\Delta x$ visualized as a geometric entity described in terms of the gradient of $f(x), \Delta x$ is seen to be unaltered by an orthogonal transformation of the coordinates $x_{1}, \cdots, x_{n}$.

Formula (2.1) is fundamental in essentially all methods employing the notion of a gradient correction in extremizing a function $f(x)$, or in solving systems of equations.

3. Description of the composite gradient method. Consider a system, written in vector form,

$$
f(x)=0,
$$

where $f(x)=\left[f_{1}(x), f_{2}(x), \cdots, f_{k}(x)\right]$ is continuously differentiable in some open convex region $\Omega$ in $n$-space. Let $f_{i j}(x)=\partial f_{j}(x) / \partial x_{i}$. Let $x$ be an assigned approximation to a solution of (3.1). Then, from (2.1), with $x^{(0)}$ replaced by $x$ in the residual $f\left(x^{(0)}\right)$, and with the part relating to the gradient taken at a point $\xi$ in $\Omega$ not necessarily the same as $x$, we write

$$
\Delta_{j} x_{i}=-f_{j}(x) f_{i j}(\xi) / w_{j}^{2}(\xi),
$$

where it is assumed that $w_{j}^{2}(x)=\sum_{i=1}^{n} f_{i j}^{2}(x) \neq 0$ in $\Omega$. In (3.2), $\xi$ is introduced to permit possible simplification in applications of the method to general systems of type (3.1). Let $\left(\eta_{1}, \cdots, \eta_{k}\right)$ be an arbitrary set of positive " weights," and define $\Delta x$ as the weighted sum of the vectors $\Delta_{j} x:$

$$
\Delta x=\sum_{j=1}^{k} \eta_{j} \Delta_{j} x .
$$

For a given approximation $x$ to a solution of (3.1), the next approximation $y$ is defined thus, where $\rho \neq 0$ is a constant whose permissible values will be discussed later:

$$
y=x+\rho \Delta x .
$$

Then (3.4) becomes the basis for a recursion formula in setting up successive approximations to a solution of (3.1), as follows.

Let $x^{(0)}$ be an initial approximation to a solution of (3.1). For $m>0$, define $x^{(m)}$ formally by the equation

$$
x^{(m)}=x^{(m-1)}+\rho^{(m-1)} \Delta x^{(m-1)},
$$


where

$$
\Delta_{j} x_{i}^{(m-1)}=-f_{j}\left(x^{(m-1)}\right) f_{i j}\left(\xi^{(m-1)}\right) / w_{j}^{2}\left(\xi^{(m-1)}\right),
$$

in which the numbers $\rho^{(m)} \neq 0, m \geq 0, \xi^{(m)}, m \geq 0$, in $\Omega$ either are designated in advance or are determined in sequence. This paper discusses mainly conditions for the existence and convergence of $\left\{x^{(m)}\right\}$, first for linear systems and later for general systems.

Note 3.1. An important special case of (3.5) and (3.6) occurs when all $\eta_{j}=1, \rho^{(m)}$ is a constant, $\rho$, for all $m$, and $\xi^{(m)}=x^{(m)}$. Then,

$$
\begin{gathered}
\Delta_{j} x_{i}^{(m-1)}=-f_{j}\left(x^{(m-1)}\right) f_{i j}\left(x^{(m-1)}\right) / w_{j}^{2}\left(x^{(m-1}\right), \\
\Delta x^{(m-1)}=\sum_{j=1}^{k} \Delta_{j} x^{(m-1)}, \quad \text { and } \quad x^{(m)}=x^{(m-1)}+\rho \Delta x^{(m-1)} .
\end{gathered}
$$

4. The linear case. In the system (not necessarily consistent)

$$
f_{j}(x) \equiv \sum_{h=1}^{n} x_{h} a_{h j}+b_{j}=0 . \quad(j=1, \cdots, k)
$$

for the unknowns $x_{h}$, let $A=\left(a_{l, j}\right)$ and $b=\left(b_{1}, \cdots, b_{k}\right)$ and rewrite (4.1) in matrix form,

$$
x A+b=0
$$

Assume that $\sum_{n=1}^{n} a_{h j}^{2} \neq 0$ for all $j$, and denote the rank of $A$ by $r$. The region $\Omega$ of $\S 3$ is taken as the whole of $n$-space. In (3.2) and (3.6), $\xi$ and $\xi^{(m-1)}$ become irrelevant.

Now let each equation in (4.1) be normalized; that is, without altering the notation, suppose that $\sum_{n=1}^{n} a_{h j}^{2}=1$, until otherwise specified. For (4.1), since the equations are normalized, (3.2) becomes

$$
\Delta_{j} x=-\left(\sum_{h=1}^{n} x_{h} a_{h j}+b_{j}\right) a_{i j} .
$$

With any assigned positive weights $\left(\eta_{1}, \cdots, \eta_{k}\right)$, let $\beta_{j}=b_{j} \eta_{j}^{1 / 2}, \alpha_{h, j}=a_{h j} \eta_{j}^{1 / 2}$, and $=\left(\alpha_{k j}\right)$. Then, from (3.3) and (4.3),

$$
\begin{aligned}
& \beta=\left(\beta_{1}, \beta_{2}, \cdots, \beta_{k}\right), \\
& \Delta x=-(x, \not+\beta) \mathscr{A}^{\prime} .
\end{aligned}
$$

If $\rho \neq 0$ is assigned, and $x$ is a designated approximation to a solution of (4.2), the next approximation, $y$, from (3.4) is $y=x+\rho \Delta x$. Before considering a sequence (3.5) for (4.2), it is desirable to recognize pro- 
perties of $\mathscr{A} \mathscr{X}^{\prime}$ which follow immediately from standard theorems, and to investigate the set $\Psi$ of points in $x$-space where $\Delta x=0$.

LEMMA 4.1. For a normalized system (4.1), if $A$ is of rank $r$, the symmetric matrix $\mathscr{A}^{\prime} \mathcal{A}^{\prime}$ is of rank $r$, is positive semidefinite, and is positive definite if and only if $r=n$. The characteristic constants of ASA consist of $n-r$ zeros and $r$ positive constants $\lambda_{1}, \cdots, \lambda_{r}$ with

$$
\sum_{i=1}^{r} \lambda_{i}=\omega, \quad \text { where } \omega=\sum_{n=1}^{n} \sum_{j=1}^{k} \alpha_{h j}^{2}=\sum_{j=1}^{k} \eta_{j} .
$$

There exists an orthogonal matrix $S=\left(s_{h m}\right)$ such that $S \mathscr{A} \mathscr{A}^{\prime} S^{\prime}=D$, where $D$ is an $n$ by $n$ diagonal matrix whose main diagonal is $\left(\lambda_{1}, \cdots, \lambda_{r}\right.$, $0, \cdots, 0)$.

The vector $\Delta x$ of (4.4) is invariant under an orthogonal transformation of coordinates in $x$-space. Thus, to obtain the set $\Psi$ where $\Delta x=0$, we first use the transformation $x=z S$ from $x$-space to $z$-space. Then with $\hat{A}=\left(\hat{a}_{h j}\right)=S A$, (4.2) becomes

$$
z \hat{A}+b=0 \text {. }
$$

LEMmA 4.2. If the equations in (4.1) are in normal form, then the equations abbreviated by (4.6) also are in normal form, that is, $\sum_{h=1}^{n} \hat{a}_{h j}^{2}=1$ for all $j$.

Proof. In $A^{\prime} A$, the $j$ th element of the main diagonal is $\sum_{n=1}^{n} a_{h j}^{2}=1$. Also

$$
\hat{A^{\prime}} \hat{A}=(S A)^{\prime} S A=A^{\prime} S^{\prime} S A=A^{\prime} I A=A^{\prime} A,
$$

where $I$ is the $n$ by $n$ unit matrix. Hence, in $\hat{A}^{\prime} \hat{A}$, the $j$ th element of the main diagonal is 1 , or $\sum_{h=1}^{n} \hat{a}_{h j}^{2}=1$.

Let $\hat{\alpha}_{h j}=\hat{\alpha}_{h j} \eta_{j}^{1 / 2}$ and $\hat{\mathscr{A}}=\left(\hat{\alpha}_{k j}\right)$. Then if $z$ is an assigned approximation to a solution of (4.6), the corresponding vector $\Delta x$ of (4.4) is given as a vector $\Delta z$, where

$$
\Delta z=-(z \hat{\mathscr{Y}}+\beta) \hat{\mathscr{\prime}}
$$

Lemma 4.3. $\hat{\mathscr{A}}=S \mathscr{A}$ and $\hat{\mathscr{A} \hat{\mathscr{A}}^{\prime}}=D$. Also, $\hat{\alpha}_{h j}=0$ if $h>r$.

Proof. 1. Since $\hat{A}=S A$,

$$
\hat{a}_{h j}=\sum_{m=1}^{n} s_{h m} a_{m j} ; \quad \hat{\alpha}_{h j}=\sum_{m=1}^{n} s_{h m}\left(a_{m j} \eta_{j}^{1 / 2}\right)=\sum_{m=1}^{n} s_{h m} \alpha_{m j} .
$$




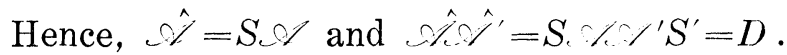

2. Since $\hat{A} \hat{A}^{\prime}=D$, where the $h$ th element of the main diagonal is zero if $h>r$, we have $\sum_{j=1}^{k} \hat{\alpha}_{h j}^{2}=0$ and hence $\hat{\alpha}_{h j}=0$ if $h>r$.

LEMmA 4.4. The solutions $\hat{z}$ of the $n$ linear equations represented by $\Delta z=0$, forming the set $\Psi$, have arbitrary $\hat{z}_{i}, i>r$, but uniquely determined $\hat{z}_{i}, i \leqq r$.

Proof. By Lemma 4.3 and (4.7), $\Delta z=0$ is equivalent to

$$
z D+\beta \mathscr{S} \hat{\nearrow}^{\prime}=0,
$$

or

$$
\left\{\begin{array}{lr}
z_{i} \lambda_{i}+\sum_{j=1}^{k} \beta_{j} \hat{\alpha}_{i j}=0, & \left(i \leqq r, \lambda_{i} \neq 0\right) \\
0+\sum_{j=1}^{k} \beta_{j} \hat{\alpha}_{i j}=0 . & (i>r)
\end{array}\right.
$$

Hence $z_{i}$ is determined uniquely if $i \leqq r$, and the equations in (4.9) for $i>r$ are identities $0=0$ because $\hat{\alpha}_{i j}=0$ for $i>r$.

Now let the origin in $z$-space be translated to any point in $\Psi$, with the new coordinates labeled $\left(u_{1}, \cdots, u_{n}\right)=u$. Then (4.6) becomes

$$
u \hat{A}+\hat{b}=0 \text {, }
$$

where $\hat{b}$ is a new one-rowed matrix of constant terms. By the method of $\S 3$, any assigned approximation $u$ to a solution of (4.10) yields a next approximation $v$ where

$$
v=u+\rho \Delta u \text { and } \Delta u=-(u \hat{\mathscr{A}}+\hat{\beta}) \hat{\mathscr{A}}^{\prime},
$$

with $\hat{\beta}=\left(\hat{b}_{1} \gamma_{1}^{1 / 2}, \cdots, \hat{b}_{k} \eta_{k}^{1 / 2}\right)$. The invariance of the composite gradient vectors $\Delta x, \Delta z, \Delta u$ under orthogonal transformations implies that $\Delta u=0$ if and only if $u$ is a point of the set $\Psi$ of Lemma 4.4. Since $u=0$ is in $\Psi, u=0$ satisfies $\Delta u=0$; thus $\hat{\beta} \hat{A}^{\prime}=$ in (4.11) and $\Delta u=0$ becomes $u D=0$, which determines $u$ as follows.

LEMMA 4.5. In the $u$-system of coordinates, we have $\Delta u=-u D$, and $\Psi$ is the set of all points $u$ for which $u_{i}=0$ if $i \leqq r$ and $u_{i}$ is arbitrary if $i>r$.

In connection with $\Psi$, consider the function ${ }^{3}$

$$
g(x)=\sum_{j=1}^{n} \eta_{j}\left(\sum_{h=1}^{n} x_{h} a_{h j}+b_{j}\right)^{2}=\|x A+\beta\|^{2} .
$$

${ }^{3}$ We shall use $\|x\|$ for the length of a vector $x,\|x\|=\left(\sum_{i=1}^{n} x_{i}^{2}\right)^{\frac{1}{2}}$. 
For any point $x, g(x)$ is the sum of weighted squares of the residuals in (4.1), with the square for the $j$ th equation given the weight $\eta_{j}$.

Lemma 4.6. The quadratic function $g(x)$ has an absolute minimum, which is attained if and only if $x$ is in $\Psi$.

Proof. The range for the function $g(x)$ is the same as the range for the corresponding function $\hat{g}(u)$, formed for (4.10), where

$$
\hat{g}(u)=\|u \hat{\mathscr{Y}}+\hat{\beta}\|^{2}=u \hat{\mathscr{A} \hat{\mathscr{Y}}} \hat{\prime}^{\prime}+2\left(\hat{\beta} \mathscr{\mathscr { A }}^{\prime}\right) u^{\prime}+\|\hat{\beta}\|^{2},
$$

or

$$
\hat{g}(u)=\sum_{h=1}^{r} \lambda_{h} u_{h}^{2}+\|\hat{\beta}\|^{2},
$$

since $\hat{\beta} \hat{\mathscr{A}}^{\prime}=0$. We see that $\hat{g}(u)$ attains its absolute minimum, $\|\hat{\beta}\|^{2}$, if and only if $u_{i}=0$ for $i \leqq r$, which describes the points in $\Psi$ and proves the lemma.

On account of Lemma 4.6, $\Psi$ may be described as the set of solutions in a sense of weighted-least-squares, for the normalized system corresponding to a system (4.1) where $\left(a_{h j}\right)$ is of rank $r$ and $\sum_{h=1}^{n} a_{h j}^{2} \neq 0$ for all $j$.

We note that $\Delta x$ of $(4.4)$ is $-\frac{1}{2} \operatorname{grad} g(x) ; \quad \Delta u=-\frac{1}{2} \operatorname{grad} \hat{g}(u)$.

THEOREM 4.1. In a system (4.1), normalized or not, where $\sum_{n=1}^{n} a_{h j}^{2} \neq 0$ for all $j$, let $r$ be the rank of $\left(a_{i, j}\right)$. Choose $\rho \neq 0$ and positive numbers $\left(\eta_{1}, \cdots, \eta_{k}\right)$ with $\omega=\sum_{j=1}^{k} \eta_{j}$. Let

$$
\zeta_{j}=\sqrt{\eta_{j}} /\left(\sum_{i=1}^{n} a_{i j}^{2}\right)^{1 / 2} ; \quad \beta=\left(b_{1} \zeta_{1}, \cdots, b_{k} \zeta_{k}\right) ; \quad \alpha_{h j}=a_{h j} \zeta_{j} ;
$$$$
\mathscr{A}=\left(\alpha_{h j}\right) \text {, with }\left(\lambda_{1}, \cdots, \lambda_{r}\right) \text { the positive }
$$
characteristic constants of $\mathscr{A}^{\prime}{ }^{\prime}$;

$$
\mu_{i}=1-\rho \lambda_{i} ; \quad \sigma_{\beta}=\max _{i \leqq r}\left|\mu_{i}\right| .
$$

For any point $x^{(0)}$ in $n$-space, let $x^{(m)}$ be defined by (3.5) when $m>0$, with $\rho^{(m)}=\rho$ for all $m \geqq 0$. Let $\hat{x}$ be the point nearest $x^{(0)}$ in the set $\Psi$ of all points $x$ where $\|x \mathscr{A}+\beta\|$ attains its absolute minimum. Then a necessary and sufficient condition that $\left\{x^{(m)}\right\}$ should converge for all points $x^{(0)}$ is that $\sigma_{\rho}<1$. Moreover, if $\sigma_{\rho}<1$, then 


$$
x^{(m)} \rightarrow \hat{x} \quad \text { and } \quad\left\|x^{(m)}-\hat{x}\right\| \leqq \sigma_{\rho}^{m}\left\|x^{(0)}-\hat{x}\right\| \cdot
$$

In order that $\sigma_{\beta}<1$,

$$
\text { it is necessary that } 0<\rho<2 r / \omega \text {; }
$$

The minimum value of $\sigma_{\rho}$ occurs, and hence the best guaranteed geometric rate of convergence is obtained in (4.15), for a single value $\rho=\rho_{0}$, where

$$
2 / \omega<\rho_{0}<2(r-1) / \omega \text { if } r>2,
$$

and

$$
\rho_{0}=r / \omega \text { if } r \leqq 2
$$

Proof. 1. The equations in (4.1) first may be normalized, and then may be altered to (4.10) in an associated $u$-system of coordinates, where $x^{(0)}$ becomes $u^{(0)}$. Application of (3.5) to (4.10), with $u^{(0)}$ as the initial point, yields a sequence $\left\{u^{(m)}\right\}$ where $u^{(m)}=u^{(m-1)}+\rho \Delta u^{(m-1)}, m>0$. The invariance property of the composite gradient corrections under orthogonal transformations justifies the statement that, in sequence, $\Delta u^{(m-1)}$ is the same vector as $\Delta x^{(m-1)}$, and hence $u^{(m)}$ is the same point as $x^{(m)}$. The matrix $\mathscr{A}$ of (4.13) may be identified with $\mathscr{A}$ in (4.4).

2. From Lemma 4.5, $\Delta u^{(m-1)}=-u^{(m-1)} D$ and

$$
u^{(m)}=u^{(m-1)}(I-\rho D), \quad \text { or } \quad u^{(m)}=u^{(0)}(I-\rho D)^{m} \quad(m>0) .
$$

In the diagonal matrix $(I-\rho D)^{m}$, the main diagonal is $\left(\mu_{1}^{m}, \cdots, \mu_{r}^{m}\right.$, $1, \cdots, 1)$, and $(4.20)$ gives

$$
u_{i}^{(m)}=u_{i}^{(0)} \mu_{i}^{m} \quad \text { if } \quad i \leqq r ; \quad u_{i}^{(m)}=u_{i}^{(0)} \quad \text { if } \quad i>r .
$$

Hence, if $\sigma_{\rho}<1, u^{(m)} \rightarrow\left(0, \cdots, 0, u_{r+1}^{(0)}, \cdots, u_{n}^{(0)}\right)$ as $m \rightarrow \infty$. In view of Lemma 4.5, this limit point is seen to be the point $\hat{u}$ of $\Psi$ nearest to $u^{(0)}$. Moreover, from (4.21).

$$
\left\|u^{(m)}-\hat{u}\right\|^{2}=\sum_{i=1}^{r} \mu_{i}^{2 m}\left[u_{i}^{(0)}\right]^{2} \leqq \sigma_{\rho}^{2 m}\left\|u^{(0)}-\hat{u}\right\|^{2} .
$$

When (4.22) is transformed back to the $x$-coordinates, the result justifies (4.15).

3. If $\sigma_{\rho}>1$, we have $\left|\mu_{i}\right|>1$, for some $i=h$. Then, if $u^{(0)}$ is selected so that $u_{h}^{(0)} \neq 0,(4.21)$ shows that $\left\{u_{h}^{(m)}\right\}$ will not converge. If $\sigma_{\rho}=1$, we have $\mu_{i}=1-\rho \lambda_{i}=1$ or -1 for some $i$. We cannot have $\mu_{i}=1$ since $\rho \lambda_{i} \neq 0$. If $\mu_{i}=-1$ for $i=h$ and $u^{(0)}$ is chosen so that $u_{h}^{(0)} \neq 0$, then $u_{h}^{(m)}$ is alternately $\pm u_{h}^{(0)}$, and $\left\{u^{(m)}\right\}$ does not converge. Thus $\left\{u^{(m)}\right\}$ does not converge for all $u^{(0)}$ if $\sigma_{\beta} \geqq 1$. 
4. To establish (4.16)-(4.19), first recall that $\sum_{i=1}^{r} \lambda_{i}=\omega$ and $\mu_{i}=1$ $-\rho \lambda_{i}$. If $\rho<0$, then $\mu_{i}>1$ for all $i$; hence, a necessary condition for $\sigma_{\rho}<1$ is that $\rho>0$, which is assumed hereafter. Let

$$
\begin{aligned}
\gamma^{\prime} & =\min _{i \leqq r} \lambda_{i} ; \quad \gamma^{\prime \prime}=\max _{i \leqq r} \lambda_{i} ; \\
\sigma^{\prime} & =\min _{i \leqq r} \mu_{i} ; \quad \sigma^{\prime \prime}=\max _{i \leqq r} \mu_{i} .
\end{aligned}
$$

Then $\sigma_{\rho}=\max \left(\left|\sigma^{\prime}\right|,\left|\sigma^{\prime \prime}\right|\right)$. The following representations on number scales summarize certain facts for the case $r \neq 1$.

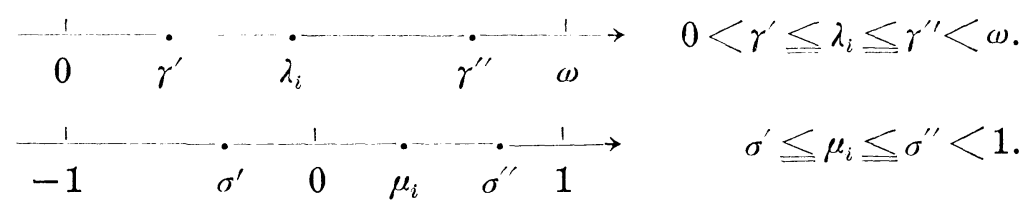

A necessary and sufficient condition for $\sigma_{\rho}<1$ is that $-1<\sigma^{\prime}$, or

$$
-1<1-\rho \gamma^{\prime \prime}, \text { or } \rho<2 / \gamma^{\prime \prime} \text {. }
$$

Since $\sum_{i=1}^{r} \lambda_{i}=\omega$ and $\lambda_{i}>0$ we have $r^{\prime \prime} \geqq \omega / r$, and (4.25) yields (4.16). If $r>1$, then $\gamma^{\prime \prime}<\omega$ and $\rho \leqq 2 / \omega$ is sufficient to imply (4.25), which establishes (4.17) if $r>1$. If $r=1$ then $\gamma^{\prime \prime}=\omega$, and the sufficient condition (4.25) becomes (4.17), as stated for $r=1$. Now suppose first that $\gamma^{\prime} \neq \gamma^{\prime \prime}$, which implies $r>1$. Then inspection of (4.24) leads to the conclusion that $\sigma_{\rho}$ attains its minimum when $\sigma^{\prime}=-\sigma^{\prime \prime}$, or

$$
\rho=2 /\left(\gamma^{\prime}+\gamma^{\prime \prime}\right) \text {. }
$$

If $r=2$, then $\gamma^{\prime}+\gamma^{\prime \prime}=\omega$ and (4.26) yields (4.19) for $r=2$. If $r>2$, we get

$$
\omega>\gamma^{\prime}+r^{\prime \prime} \geqq \frac{\omega-r^{\prime}}{r-1}+r^{\prime}>\frac{\omega}{r-1} .
$$

From (4.26) and (4.27), (4.18) is obtained. If $\gamma^{\prime}=\gamma^{\prime \prime}$, then $\sigma_{\rho}=|1-\rho \omega| r \mid$, whose minimum is 0 , attained at $\rho=r / \omega$, which agrees with (4.18) if $r>2$ and with (4.19) if $r \leqq 2$. Hence, all cases of (4.16)-(4.19) have been justified, which concludes the proof of Theorem 4.1.

CoROLlaRY 4.1. The geometric rate of convergence indicated in (4.15) is the fastest rate of convergence holding for the method in general.

Proof. Consider a system (4.1) where $b_{j}=0$ and $n=k=r=2$, so that $x=0$ is the unique solution. Let $\rho=\eta_{1}=\eta_{2}=1$. Let $x^{(0)}$ be any point $\neq 0$; then Theorem 4.1 states that $x^{(m)}$ of $(4.15)$, or $u^{(m)}$ or $(4.20)$, 
converges to 0 . In this case,

$$
\lambda_{1}+\lambda_{2}=2 \text { and } \mu_{1}+\mu_{2}=2-\left(\lambda_{1}+\lambda_{2}\right)=0 .
$$

Hence we may let $\mu_{1}=\mu>0$ and $\mu_{2}=-\mu$, with $\sigma_{p}=\mu<1$. Then (4.21) gives $u_{1}^{(m)}=u_{1}^{(0)} \mu^{m}$ and $u_{2}^{(m)}=u_{2}^{(0)}(-\mu)^{m}$. With $\hat{u}=0$ in (4.22),

$$
\left\|u^{(m)}-\hat{u}\right\|^{2}=\mu^{2 m}\left\|u^{(0)}-\hat{u}\right\|^{2}, \quad \text { or } \quad\left\|u^{(m)}-\hat{u}\right\|=\sigma_{\rho}^{m}\left\|u^{(0)}-\hat{u}\right\| \text {. }
$$

Thus the equality sign applies in (4.15) and the corollary is proved.

Note 4.1. Consider altering the method of Theorem 4.1 by introducing $\rho^{(m)}$ as in (3.5), with $0<\rho^{(m)}<2 r / \omega$. Let all pertinent notation from Theorem 4.1 be used, with (4.14) replaced by

$$
\mu_{i}^{(m)}=1-\rho^{(m)} \lambda_{i} ; \quad \sigma^{(m)}=\max _{i \leqq r}\left|\mu_{i}^{(m)}\right| ; \quad \sigma=\sup _{\text {all } m} \sigma^{(m)} .
$$

Then, if $\sigma<1$, it is found that (4.15) holds with $\sigma_{\rho}$ replaced by $\sigma$. Also, in order that $\sigma<1$, it is sufficient that $0<\rho^{(m)} \leqq 2 / \omega$ if $r>1$ and $\rho^{(m)} \leqq \rho<2 / \omega$ if $r=1$.

The following result will be used later.

Corollary 4.2, In a system (4.1), with the equations in the normal form, suppose that $A$ is of rank $n$ and that (4.1) has a solution $\hat{x}$, necessarily unique. Let $x$ be an assigned approximation to $\hat{x}$ with $y$ as the next approximation, where $\Delta x$ is given by (4.4) and $y=x+\rho \Delta x$. Then

$$
\Delta x=-(x-\hat{x}) \mathscr{C O}^{\prime} ; y-\hat{x}=(x-\hat{x}) B_{\rho} \quad \text { where } B_{\rho}=I-\rho \mathscr{C A} \mathcal{L}^{\prime} ;
$$

$$
\|y-\hat{x}\|^{2}=(x-\hat{x}) B_{\rho}^{2}(x-\hat{x})^{\prime} \leqq \sigma_{\rho}^{2}\|x-\hat{x}\|^{2} .
$$

Proof. In this case, (4.2) can be written $(x-\hat{x}) A=0$, and (4.4) gives $\Delta x=-(x-\hat{x}) / \mathscr{L}^{\prime} ; y-\hat{x}=(x-\hat{x}) B_{\rho}$. Then from (4.15) with $m=1$, $x^{(1)}=y$, and $x^{(0)}=x$, we obtain (4.29).

5. Solution of a general system. Consider the system (3.1), or

$$
f_{j}(x)=0 \quad(j=1, \cdots, k) .
$$

Assume that $\hat{x}$ is a solution of (5.1) and that, for all $i$ and $j, f_{i j}(x)$ is continuous and $w_{j}^{2}(x) \neq 0$ in some open convex neighborhood $\Omega$ of $x=\hat{x}$. All points $x$ will be restricted to $\Omega$. At $x=\hat{x}$, the surface $f_{j}(x)=0$ has a tangent plane $\sum_{h=1}^{n} a_{h, j}\left(x_{h}-\hat{x}_{h}\right)=0$, where $a_{h j}=f_{h j}(\hat{x})$. Assume that the matrix $A=\left(a_{h j}\right)$ has rank $n$, which implies that $k \geqq n$, and that the tangent planes to the surfaces $f_{j}(x)=0$ at $x=\hat{x}$ have the unique intersection $x=\hat{x}$. Then the method of $\S 3$ will be applied to obtain $\hat{x}$ as the limit of a sequence $\left\{x^{(m)}\right\}$, defined in (3.5). 
Let $c_{i j}(x)=f_{i j}(x) / w_{j}^{2}(x) ; C(x)=\left(c_{i j}(x)\right) ; F(x)=\left(f_{i j}(x)\right)$. Let $x$ be an assigned approximation to a solution of (5.1), and let $\xi$ be any point in $\Omega$. Then (3.2) and (3.3) give

$$
\Delta_{j} x_{i}=-f_{j}(x) c_{i j}(\xi) ; \quad \Delta x=\sum_{j=1}^{k} \eta_{j} \Delta_{j} x .
$$

With $\rho>0$ and $\eta_{3}>0$, the next approximation $y$ to a solution of (5.1) is $y=x+\rho \Delta x$.

For given $A$ and weights $\eta_{j}$, let $\sigma_{\rho}$ have the same meaning as in Theorem 4.1.

Lemma 5.1. Suppose that $\sigma_{p} \leqq \sigma<1$. Then, for every $\theta$ such that $\sigma<\theta<1$, there exists a $\delta>0$ such that the neighborhood $\Phi_{o}:(\|x-\hat{x}\|<\delta)$ is in $\Omega$ and, if $x$ is in $\Phi_{\delta}, \xi$ is in $\Phi_{\delta}$, and $y=x+\rho \Delta x$, then

$$
\|y-\hat{x}\| \leqq \theta\|x-\hat{x}\| \text {. }
$$

Proof. 1. For convenience, without change of notation, suppose that both sides of $f_{j}(x)=0$ have been multiplied by a proper constant so that $w_{j}^{2}(\hat{x})=1$, for all $j$. Then $C(\hat{x})=F(\hat{x})=A$.

2. By the mean value theorem, since $f(\hat{x})=0$,

$$
f_{j}(x)=\sum_{h=1}^{n}\left(x_{h}-\hat{x}_{h}\right) a_{h j}+\sum_{h=1}^{n}\left(x_{h}-\hat{x}_{h}\right) p_{h j}(x),
$$

where $p_{h j}(x)=f_{h j}\left(\tau_{j}\right)-f_{h j}(\hat{x})$ and $\tau_{j}$ is suitably located on the line segment from $\hat{x}$ to $x$. Let

$$
\begin{array}{cc}
\alpha_{h j}=a_{h j} \eta_{j}^{1 / 2} ; \quad \pi_{h j}(x)=p_{h j}(x) \eta_{j}^{1 / 2} ; \quad & \gamma_{h j}(x)=c_{h j}(x) \eta_{j}^{1 / 2} ; \\
& v_{h j}(x)=\gamma_{h j}(x)-\alpha_{h j} ; \\
A=\left(\alpha_{h j}\right) ; \quad P(x)=\left(\pi_{h j}(x)\right) ; \quad V(x)=\left(v_{h j}(x)\right) .
\end{array}
$$

Note that $\gamma_{h j}(\hat{x})=\alpha_{h j} ; p_{h j}(x) \rightarrow 0$ and $v_{h, j}(x) \rightarrow 0$ if $x \rightarrow \hat{x}$. From (5.2) and (5.4), we find

$$
\Delta x=-(x-\hat{x})\left(\mathscr{Z}+P(x)(\mathscr{Z}+V(\xi))^{\prime} .\right.
$$

3. Since $y-\hat{x}=(x-\hat{x})+\rho \Delta x$, we have

$$
y-\hat{x}=(x-\hat{x}) B_{\rho}+(x-\hat{x}) Q(x, \xi),
$$

where $B_{\rho}=I-\rho^{\prime} \chi^{\prime}$ and

$$
Q(x, \xi)=-\rho \mathscr{\nearrow} V^{\prime}(\xi)-\rho P(x) \mathscr{Z}^{\prime}-\rho P(x) V^{\prime}(\xi) .
$$

Вy (5.5),

$$
\|y-\hat{x}\|^{2}=(x-\hat{x}) B_{\rho}^{2}(x-\hat{x})^{\prime}+(x-\hat{x}) U(x, \xi)(x-\hat{x})^{\prime},
$$


where

$$
U(x, \xi)=B_{\rho} Q^{\prime}(x, \xi)+Q(x, \xi) B_{\rho}+Q(x, \xi) Q^{\prime}(x, \xi),
$$

and we let $U(x, \xi)=\left(u_{i,}(x, \xi)\right)$. By use of the Cauchy inequality twice ${ }^{t}$

$$
\left|(x-\hat{x}) U(x, \xi)(x-\hat{x})^{\prime}\right| \leqq\|x-\hat{x}\|^{2}\left[\sum_{i, j=1}^{n} u_{i j}^{2}(x, \xi)\right]^{1 / 2} .
$$

Then (4.29) and (5.7) yield

$$
\|y-\hat{x}\|^{2} \leqq\|x-\hat{x}\|^{2}\left\{\sigma_{\rho}^{2}+\left[\sum_{i, j=1}^{n} u_{i j}^{2}(x, \xi)\right]^{1 / 2}\right\} .
$$

4. In (5.8), $u_{i j}(x, \xi)$ is a polynomial in the elements $p_{i j,}(x)$ and $v_{h j}(\xi)$, with each term of the polynomial of degree 1 at least in the elements. Hence $u_{i_{2}}(x, \xi) \rightarrow 0$ as $x \rightarrow \hat{x}$ and $\xi \rightarrow \hat{x}$. Now suppose that $\sigma_{\beta} \leqq \sigma<1$, and choose $\theta$ so that $\sigma<\theta<1$. From (5.8), if $\delta$ is chosen so that the neighborbood $\Phi_{\delta}:(\|x-\hat{x}\|<\delta)$ is in $\Omega$, and if $\delta$ also is sufficiently small, then $\|y-\hat{x}\|^{2} \leqq \theta^{2}\|x-\hat{x}\|^{2}$ if $x$ is in $\Phi_{\delta}$ and $\xi$ is in $\Phi_{\delta}$, which proves the lemma.

Theorem 5.1. Assume that (5.1) has the solution $x=\hat{x}$, that $F(\hat{x})$ is of rank $n$, and $w_{j}^{2}(x) \neq 0$ in $\Omega$. Then, if the $\rho^{(m)}$ are properly chosen, with $0<\rho^{(m)}<2 n / \omega$, there exists $a \theta>0$, with $\theta<1$, and a corresponding $\delta>0$ so that, if $x^{(0)}$ and the $\xi^{(m)}$ are arbitrary points in $\Phi_{\delta}$ : $(\|x-\hat{x}\|<\delta)$, and $x^{(m)}$ is given by (3.5), then $x^{(m)} \rightarrow \hat{x}$ as $m \rightarrow \infty$, in such a manner that

$$
\left\|x^{(m)}-\hat{x}\right\| \leqq \theta^{m}\left\|x^{(0)}-\hat{x}\right\| .
$$

It is sufficient to use a constant $\rho^{(m)}=\rho$ where $\rho \leqq 2 / \omega$ if $n>1$ and $\rho<$ $2 / \omega$ if $n=1$. Moreover ${ }^{5}, \hat{x}$ is the unique solution of (5.1) in $\Phi_{\delta}$.

Proof. 1. Let $\sigma^{(m)}=\sigma_{\beta}$ for $\rho=\rho^{(m)}$. Let the $\rho^{(m)}$ be chosen so that $\sup _{\text {all } m} \sigma^{(m)}=\sigma<1$. In particular, by (4.17), the preceding condition is satisfied if all $\rho^{(m)}=2 / \omega$ if $n>1$ and if $\rho^{(m)}=\rho<2 / \omega$ if $n=1$. Now choose $\theta$ so that $\sigma<\theta<1$, and let $\delta>0$ be determined by Lemma 5.1. Select $x^{(0)}$ and the sequence $\xi^{(m)}$ arbitrarily in $\Phi_{\dot{\delta}}$. Then, from Lemma 5.1,

$$
\left\|x^{(1)}-\hat{x}\right\| \leqq \theta\left\|x^{(0)}-\hat{x}\right\|<\delta ; \quad\left\|x^{(2)}-\hat{x}\right\| \leqq \theta\left\|x^{(1)}-\hat{x}\right\| \leqq \theta^{2}\left\|x^{(0)}-\hat{x}\right\|<\delta \text {; etc. }
$$

It follows that all $x^{(m)}$ of (3.5) are in $\Phi_{\delta}$ and satisfy (5.9). Hence $x^{(m)} \rightarrow \hat{x}$ as $m \rightarrow \infty$.

${ }^{4}$ As follows:

$\left[\sum_{i=1}^{n} z_{i}\left(\sum_{j=1}^{n} u_{i j} z_{j}\right)\right]^{2} \leqslant \sum_{i=1}^{n} z_{i}^{2} \sum_{i=1}^{n}\left(\sum_{j=1}^{n} u_{i j} z_{j}\right)^{2} \leq\left(\sum_{i=1}^{n} z_{i}^{2}\right)^{2} \sum_{i, j=1}^{n} u_{i j}^{2}$.

5 For a different condition assuring uniqueness, see [5]. 
2. Suppose that a point $z \neq \hat{x}$ exists in $\Phi_{\delta}$ where $f(\hat{z})=0$. If we choose $x^{(0)}=z$ in (3.5), then $x^{(1)}=z$ and (5.9) gives $\|z-\hat{x}\| \leqq \theta\|z-\hat{x}\|<$ $\|z-\hat{x}\|$, which is a contradiction. Hence $\hat{x}$ is the only solution of (5.1) in $\Phi_{s}$.

CoROllary 5.1. There exists a best constant $\rho^{(m)}=\rho_{0}$ for the method of Theorem 5.1, in the sense that this choice for $\rho^{(m)}$ allows the smallest lower bound for $\theta$ in (5.9), with

$$
\begin{gathered}
2 / \omega<\rho_{0}<2(n-1) / \omega \text { if } n>2 ; \\
\rho_{0}=n / \omega \text { if } n \leqq 2 .
\end{gathered}
$$

Proof. With $\rho^{(m)}=\rho_{0}$ as specified by (4.18) and (4.19) with $r=n$, the minimum value for $\sigma$ is obtained in the proof of Theorem 5.1. Since $\sigma=\inf \theta$, the corollary is established.

Note 5.1. The presence of $\left\{\rho^{(m)}\right\}$ and $\left\{\xi^{(m)}\right\}$ in the definition of the sequence $\left\{x^{(m)}\right\}$ is designed to permit latitude in the computational use of the method to solve a system (5.1), particularly by means of a high speed digital computing machine. In any application, the process would start with an approximation $x^{(0)}$ which is hoped to be close enough to the unknown solution. Probably it would be sensible to use $\xi^{(m)}=x^{(m)}$ until $\left\|f\left(x^{(m)}\right)\right\|$ becomes small and changes become regular in all variables, so that there is evidence of future success for the process. Thereafter $\xi^{(m)}$ might be changed only periodically, say at intervals of $s$ iterations, instead of at each step. Also, breakpoints might be introduced in the coding for machine calculation to permit trial of various values of $\rho^{(m)}$, perpaps starting with the safe value $\rho^{(0)}=2 / \omega$ when $n>1$. In practice it has been found that convergence to the desired solution can be accelerated if, periodically, preceding values of $x^{(m)}$ are taken as a basis for extrapolation, after the process has brought $x^{(m)}$ close enough to a solution to create smoothly changing increments $\Delta x^{(m)}$.

6. Small arc computation of an implicit function. Consider the system

$$
g(x ; \tau)=0,
$$

or $g_{j}(x ; \tau)=0, j=1, \cdots, n$, where $\tau=\left(\tau_{1}, \cdots, \tau_{k}\right)$ and $(\hat{x} ; \hat{\tau})$ is a solution. Assume that all derivatives $g_{i j}=\partial g_{j} / \partial x_{i}$ exist and are continuous in a closed region $\Phi:(\|x-\hat{x}\| \leqq a,\|\tau-\hat{\tau}\| \leqq b)$, and that the Jacobian $J=\partial\left(g_{1}\right.$, $\left.\cdots, g_{n}\right) / \partial\left(x_{1}, \cdots, x_{n}\right) \neq 0$ in $\Phi$. Then, the classical theorem on implicit functions (for example, [3, p. 138]) states that positive numbers $\varepsilon<a$ 
and $\gamma<b$ exist such that, if $\|\tau-\hat{\tau}\|<\gamma$, there is an unique point $\tilde{x}(\tau)$ with $\|\tilde{x}(\tau)-\hat{x}\|<\varepsilon$ for which $g[\tilde{x}(\tau) ; \tau]=0$. Moreover, $\tilde{x}(\tau)$ is a continuous function of $\tau$ in the neighborhood $\|\tau-\hat{\tau}\|<\delta$. Let an arc $\Lambda$ through $\hat{\tau}$ in $\tau$-space be defined by $\tau=\tau(t), 0 \leqq t \leqq 1$, where $\tau(0)=\hat{\tau},\|\tau(t)-\hat{\tau}\|<$ $\gamma$, and all $\tau,(t)$ are continuous. At each value of $t$, there is a solution $x(t)=\tilde{x}[\tau(t)]$ for (6.1) with $\tau=\tau(t)$ in (6.1). Assume that the solution $(\hat{x} ; \hat{\tau})$ is known. Then we shall consider the problem of computing $x(t)$ for a finite number of points on $\Lambda$ extending from $t=0$ to $t=1$.

Transform (6.1) by substituting $\tau=\tau(t)$ and dividing both sides of the $j$ th equation by $\left\{\sum_{i=1}^{n} g_{i,}^{2}[x(t) ; \tau(t)]\right\}^{1 / 2}$. Then (6.1) becomes

$$
f_{j}(x ; t)=0, \quad(j=1, \cdots, n ; 0 \leqq t \leqq 1)
$$

which has the unique solution $x=x(t)$ in the neighborhood $\|x-\hat{x}\|<\varepsilon$. Let $c_{i j}(x ; t)=f_{i j}(x ; t) / w_{j}^{2}(x ; t)$, where $f_{i j}=\partial f_{1} / \partial x_{i}$. Notice that $w_{j}^{2}(x ; t)$ $=\sum_{i=1}^{n} f_{i j}^{2}(x ; t)$ is bounded from zero in the closed region $\hat{\Phi:}(\|x-\hat{x}\| \leqq a$, $0 \leqq t \leqq 1$ ) because the Jacobian $J \neq 0$ in $\Phi$. Let

$$
\begin{aligned}
& C(x ; t)=\left(c_{i j}(x ; t)\right) ; \quad F(x ; i)=\left(f_{i \jmath}(x ; t)\right) ; \\
& A(t)=F[x(t) ; t]=\left(a_{i j}(t)\right),
\end{aligned}
$$

where $A(t)$ is nonsingular because $J \neq 0$ in $\Phi$. Also, each column of $A(t)$ is a vector with length 1 because of the normalization in (6.2). Thus, for each value of $t, A(t)$ satisfies the conditions imposed on $A$ in $\S 4$, with $n=k=r$ and each column of $A(t)$ normalized. For simplicity choose the weights $\eta_{j}=1$, and then let $\sigma_{\rho}(t)$ have the meaning, for $A(t)$, of $\sigma_{\rho}$ for $A$ in Theorem 4.1, where we now have $\omega=n$.

Lemma 6.1. There exist values of $\rho$, in particular $\rho=2 / n$ if $n>1$ and any $\rho<2 / n$ if $n=1$, such that

$$
\max _{0 \leqq t \leqq 1} \sigma_{\rho}(t)=\hat{\sigma}_{\rho}<1 .
$$

Proof. The characteristic constants $\lambda_{i}(t)$ of $A(t) A^{\prime}(t)$ are continuous and hence $\sigma_{\rho}(t)$ is continuous. With the particular values specified for $\rho$ in the lemma, $\sigma_{\rho}(t)<1$ for $0 \leqq t \leqq 1$ and hence $\hat{\sigma}_{\rho}<1$.

Since $\|x(t)-\hat{x}\|<\varepsilon$ for $0 \leqq t \leqq 1$ and $x(t)$ is continuous, we obtain the following result.

LEMMA 6.2. There exists a $\zeta>0$ such that, if $\|x-x(t)\|<\zeta$ for some $t$, then $\|x-\hat{x}\|<\varepsilon$.

If $\|x-\hat{x}\|<a$, we may expand each $f_{\jmath}(x ; t)$ by the mean value 
theorem, as in (5.4), with respect to the variables $x_{i}$ at $x=x(t)$; since $f[x(t) ; t]=0$,

$$
f(x ; t)=[x-x(t)][A(t)+P(x ; t)],
$$

where $P(x ; t)=\left(p_{i},(x ; t)\right)$. Observe that $p_{i \jmath}(x ; t) \rightarrow 0$ as $\|x-x(t)\| \rightarrow 0$, uniformly for $0 \leqq t \leqq 1$, because $\hat{\Phi}$ is a closed region.

Let $v_{i,}(x ; t)=c_{i j}(x ; t)-c_{i, 2}[x(t) ; t]$, and $V(x ; t)=\left(v_{i, 2}(x ; t)\right)$. Since $w_{\jmath}^{2}(x ; t)$ is bounded from zero in $\hat{\Phi}, c_{i \jmath}(x ; t)$ is continuous in $\hat{\phi}$. Hence $v_{i,}(x ; t) \rightarrow 0$ as $\|x-x(t)\| \rightarrow 0$, uniformly for $0 \leqq t \leqq 1$. Since $c_{i j}[x(t) ; t]$ $=a_{i j}(t)$,

$$
C(x ; t)=A(t)+V(x ; t) .
$$

LemMa 6.3. Let $t$ be fixed, let $\zeta$ be specified by Lemma 6.2, and let $x$ be an assigned approximation to $x(t)$, with $\|x-x(t)\|<\zeta$. Define a next approximation to $x(t)$ by

$$
y=x+\rho \Delta x, \text { where } \Delta x=-f(x ; t) C^{\prime}(x ; t),
$$

and $\hat{\sigma}_{\beta}<1$. Then, for every $\theta$ such that $\hat{\sigma}_{\rho}<\theta<1$, there exists a $\delta$, $0<\delta \leqq \zeta$, where $\delta$ is independent of $t$, such that

$$
\|x-x(t)\|<\delta \text { implies that }\|y-x(t)\| \leqq \theta\|x-x(t)\| .
$$

A proof of Lemma 6.3 would duplicate details in the proof of Lemma 5.1, with dependence on $t$ introduced, and with $\hat{x}=x(t), \xi=x$, and $\sigma=\hat{\sigma}_{\beta}$. Limits as $\|x-\hat{x}\| \rightarrow 0$ in the proof of Lemma 5.1 would be replaced by limits as $\|x-x(t)\| \rightarrow 0$, and these limits exist uniformly for $0 \leqq t \leqq 1$. Thus (6.4) is derived with $\delta$ independent of $t$.

Theorem 6.1. Let $t_{0}, t_{1}, \cdots, t_{l}$ be such that $0=t_{0}<t_{1}<\cdots<t_{l}=1$. Let $x^{(0)}\left(t_{i}\right)=x\left(t_{i-1}\right), i>0$, choose $\rho$ so that $\hat{\sigma}_{\rho}<1$, and define

$$
x^{(m)}\left(t_{i}\right)=x^{(m-1)}\left(t_{i}\right)-\rho f\left[x^{(m-1)}\left(t_{i}\right) ; t_{i}\right] C^{\prime}\left[x^{(m-1)}\left(t_{i}\right) ; t_{i}\right] .
$$

Then, for any $\theta$ such that $\hat{\sigma}_{p}<\theta<1$, there exists $a \psi>0$ such that, if $t_{i}-t_{i-1}<\psi$, then $x^{(m)}\left(t_{i}\right) \rightarrow x\left(t_{i}\right)$ as $m \rightarrow \infty$, for $i=1, \cdots, l$ and

$$
\left\|x^{(m)}\left(t_{i}\right)-x\left(t_{i}\right)\right\| \leqq \theta^{m}\left\|x\left(t_{i-1}\right)-x\left(t_{i}\right)\right\| .
$$

Proof. If $\theta$ is such that $\hat{o}_{\rho}<\theta<1$, Lemma 6.3 specifies a $\delta>0$ for which (6.4) is true. Since $x(t)$ is continuous, there exists a $\psi>0$ such that $\left\|x\left(t^{\prime \prime}\right)-x\left(t^{\prime}\right)\right\|<\delta$ if $\left|t^{\prime \prime}-t^{\prime}\right|<\psi$. Then, by Lemma 6.3, if $t_{i}-t_{i-1}<\psi$ for all $i>0$, it can be verified, along the lines of the proof of Theorem 5.1, that all $x^{(m)}\left(t_{2}\right)$ are well defined and satisfy (6.6), which proves Theorem 6.1 . 
The normalization involved in passing from (6.1) to (6.2) has no effect on gradient corrections, as met in (6.5). Hence the following "small arc method" for computing values of the implicit function $\tilde{x}(t)$ given by a system (6.1) emerges from Theorem 6.1.

For some $\tau=\hat{\tau}$, suppose that the solution $x=\hat{x}$ of (6.1) is known. Let $\tau=\tau(t), 0 \leqq t \leqq 1$, be a particular continuous curve through $\tau=\hat{\tau}=$ $\tau(0)$. Substitute $\tau=\tau(t)$ in (6.1) to obtain $\tilde{g}(x ; t)=0$, with the solution $x=x(t)$. Choose $\rho=2 / n$ if $n>1$, and refer to our composite gradient method in Theorem 5.1, with $\rho^{(m)}=2 / n, \xi^{(m)}=x^{(m)}$, and $\eta_{3}=1$ for simplicity here ${ }^{6}$. Select a partition $t_{0}, t_{1}, \cdots, t_{l}$ of the interval $0 \leqq t \leqq 1$, with all $t_{i}-t_{i-1}$ as small as necessary, where the decision as to size is based on computing sense, supported experimentally by later details of computation. Then determine $x\left(t_{1}\right)$ as the solution of $\tilde{g}\left(x ; t_{1}\right)=0$ by our gradient method, with $x^{(0)}\left(t_{1}\right)=x(0)=\hat{x}$; determine $x\left(t_{i}\right), i>0$, by using $x^{(0)}\left(t_{l}\right)=x\left(t_{i-1}\right)$. In the preceding sentence, "determine" is interpreted as " obtain exactly," for logical application of Theorem 6.1. However, in any numerical example, "determine" would mean "find accurately to the degree of precision specified by the problem, and necessary for the remainder of the procedure."

Note 6.1. Consider solving a given system of $n$ algebraic equations in $x$, with constant coefficients $\left(\alpha_{1}, \cdots, \alpha_{k}\right)=\tau$. Suppose that the solution of the system is known when $\tau=\hat{\tau}$. Then, the small arc method might be used to obtain the solution $x$ when $\tau=\alpha$, by employing the line segment $\tau=\tau(t)=\hat{\tau}+(\alpha-\hat{\tau}) t, 0 \leqq t \leqq 1$, or any suitable continuous curve joining $\tau=\hat{\tau}$ and $\tau=\alpha$.

\section{REFERENCES}

1. Herman Chernoff and Jean Bronfenbrenner Crockett, Gradient methods of maximization, Pacific J. Math., 5 (1955), 33-50.

2. George E. Forsythe, Solving linear equations can be interesting, Bull. Amer. Math. Soc., 59 (1953), 299-329.

3. Lawrence M. Graves, The theory of functions of real variables, McGraw Hill, 1946.

4. Theodore S. Motzkin and C. B. Tompkins, Boundedness of sequential projections (abstract), Bull. Amer. Math. Soc., 59 (1953), 396.

5. Alexandre Ostrowski, Un Théorème d'existence pour les systèmes d'équations, C. R. Acad. Paris, 231 (1950), 1114-1116.

6. C. B. Tompkins, Projection mothods in calculation, Proceedings of the Second Symposium in Linear Programming, Washington D. C., 2 (1955), 425-448.

UNIVERSITY OF MINNESOTA,

University of California, Los Angeles

6 Weights $\eta_{j} \neq 1$ and points $\xi^{(m)} \neq x^{(m)}$ could be employed. 



\section{PACIFIC JOURNAL OF MATHEMATICS}

\section{EDITORS}

H. L. Royden

Stanford University

Stanford, California

E. Hewits

University of Washington

Seattle 5 , Washington
R. P. Dilworth

California Institute of Technology Pasadena 4, California

E. G. Straus

University of California

Los Angeles 24, California

\section{ASSOCIATE EDITORS}
E. F. BECKENBACH
C. E. BURGESS
H. BUSEMANN
H. FEDERER

\author{
M. HALL \\ P. R. HALMOS \\ V. GANAPATHY IYER \\ R. D. JAMES
}

\author{
M. S. KNEBELMAN \\ I. NIVEN \\ T. G. OSTROM \\ M. M. SCHIFFER
}

\section{J. J. STOKER \\ G. SZEKERES \\ F. WOLF \\ K. YOSIDA}

\section{SUPPORTING INSTITUTIONS}

UNIVERSITY OF BRITISH COLUMBIA CALIFORNIA INSTITUTE OF TECHNOLOGY

UNIVERSITY OF CALIFORNIA

MONTANA STATE UNIVERSITY

UNIVERSITY OF NEVADA

OREGON STATE COLLEGE

UNIVERSITY OF OREGON

UNIVERSITY OF SOUTHERN CALIFORNIA
STANFORD UNIVERSITY

UNIVERSITY OF UTAH

WASHINGTON STATE COLLEGE

UNIVERSITY OF WASHINGTON

$*$ * * *

AMERICAN MATHEMATICAL SOCIETY

CALIFORNIA RESEARCH CORPORATION HUGHES AIRCRAFT COMPANY

Mathematical papers intended for publication in the Pacific Journal of Mathematics should be typewritten (double spaced), and the author should keep a complete copy. Manuscripts may be sent to any of the editors. Manuscripts intended for the outgoing editors should be sent to their successors. All other communications to the editors should be addressed to the managing editor, E. G. Straus at the University of California, Los Angeles 24, California.

50 reprints of each article are furnished free of charge; additional copies may be obtained at cost in multiples of 50 .

The Pacific Journal of Mathematics is published quarterly, in March, June, September, and December. The price per volume (4 numbers) is $\$ 12.00$; single issues, $\$ 3.50$. Back numbers are available. Special price to individual faculty members of supporting institutions and to individual members of the American Mathematical Society: $\$ 4.00$ per volume; single issues, $\$ 1.25$.

Subscriptions, orders for back numbers, and changes of address should be sent to Pacific Journal of Mathematics, 2120 Oxford Street, Berkeley 4, California.

Printed at Kokusai Bunken Insatsusha (International Academic Printing Co., Ltd.), No. 10, 1-chome, Fujimi-cho, Chiyoda-ku, Tokyo, Japan.

\section{PUBLISHED BY PACIFIC JOURNAL OF MATHEMATICS, A NON-PROFIT CORPORATION}

The Supporting Institutions listed above contribute to the cost of publication of this Journal, but they are not owners or publishers and have no responsibility for its content or policies. 


\section{Pacific Journal of Mathematics}

\section{Vol. 6, No. 4}

1956

Seymour Ginsburg, On mappings from the family of well ordered subsets of

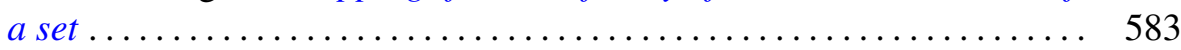

Leon Ehrenpreis, Some properties of distributions on Lie groups ......... 591

Marion K. Fort, Jr., A geometric problem of Sherman Stein ............. 607

Paul R. Garabedian, Calculation of axially symmetric cavities and jets . . . . 611

Walter Mossman Gilbert, Completely monotonic functions on cones ...... 685

William L. Hart and T. S. Motzkin, A composite Newton-Raphson gradient method for the solution of systems of equations ................. 691

C. W. Mendel and I. A. Barnett, A functional independence theorem for square matrices ................................. 709

Howard Ashley Osborn, The problem of continuous programs .......... 721

William T. Reid, Oscillation criteria for linear differential systems with complex coefficients ............................. 733

Irma Reiner, On the two-adic density of representations by quadratic

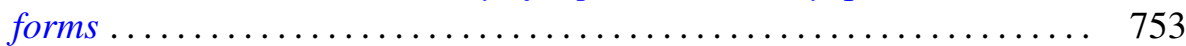

Shoichiro Sakai, A characterization of $W^{*}$-algebras .............. 763

Robert Steinberg, Note on a theorem of Hadwiger................. 775

$\mathrm{J}$. Eldon Whitesitt, Construction of the lattice of complemented ideals within

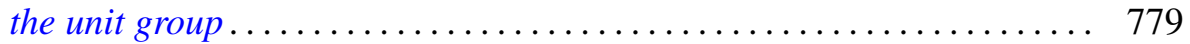

Paul Civin, Correction to "Some ergodic theorems involving two operators"... 